\title{
Unilateral maxillary central incisor root resorption after orthodontic treatment for Angle Class 11, division 1 malocclusion with significant maxillary midline deviation: A possible correlation with root proximity to the incisive canal
}

Toshihiro Imamura (10) Shunsuke Uesugi

Takashi Ono

Department of Orthodontic Science, Graduate School of Medical and Dental Sciences, Tokyo Medical and Dental University, Tokyo, Japan
Root resorption can be caused by several factors, including contact with the cortical bone. Here we report a case involving a 21-year-old female with Angle Class Il, division 1 malocclusion who exhibited significant root resorption in the maxillary right central incisor after orthodontic treatment. The patient presented with significant left-sided deviation of the maxillary incisors due to lingual dislocation of the left lateral incisor and a Class 11 molar relationship. Cephalometric analysis demonstrated a Class 1 skeletal relationship (A pointnasion-B point, $2.5^{\circ}$ ) and proclined maxillary anterior teeth (upper incisor to sella-nasion plane angle, $113.4^{\circ}$ ). The primary treatment objectives were the achievement of stable occlusion with midline agreement between the maxillary and mandibular dentitions and appropriate maxillary anterior tooth axes and molar relationship. A panoramic radiograph obtained after active treatment showed significant root resorption in the maxillary right central incisor; therefore, we performed cone-beam computed tomography, which confirmed root resorption along the cortical bone around the incisive canal. The findings from this case, where different degrees of root resorption were observed despite comparable degrees of orthodontic movement in the bilateral maxillary central incisors, suggest that the incisive canal could be an inducing factor for root resorption. However, further investigation is necessary to confirm this assumption.

[Korean J Orthod 2020;50(3):216-226]

Key words: Root resorption, Computed tomography, Digital models, Incisive canal Received December 24, 2018; Revised June 4, 2019; Accepted June 14, 2019.

Corresponding author: Toshihiro lmamura.

Postdoctoral Fellow, Department of Orthodontic Science, Graduate School of Medical and Dental Sciences, Tokyo Medical and Dental University, 1-5-45 Yushima, Bunkyo-ku, Tokyo 113-8549, Japan.

Tel +81-3-5803-5529 e-mail imamura.orts@gmail.com

How to cite this article: Imamura T, Uesugi S, Ono T. Unilateral maxillary central incisor root resorption after orthodontic treatment for Angle Class 11, division 1 malocclusion with significant maxillary midline deviation: A possible correlation with root proximity to the incisive canal. Korean J Orthod 2020;50:216-226.

(C) 2020 The Korean Association of Orthodontists.

This is an Open Access article distributed under the terms of the Creative Commons Attribution Non-Commercial License (http://creativecommons.org/licenses/by-nc/4.0) which permits unrestricted non-commercial use, distribution, and reproduction in any medium, provided the original work is properly cited. 


\section{INTRODUCTION}

Cases of Angle Class 11, division 1 malocclusion often show root resorption in the maxillary anterior teeth after orthodontic treatment. ${ }^{1}$ In recent years, the innovation of implant anchorage systems has enabled a large amount of maxillary anterior tooth retraction, ${ }^{2}$ which in turn has increased the risk of root resorption in the maxillary anterior teeth. There are various contributing factors for root resorption, including root proximity to the cortical bone. ${ }^{3,4}$ In the maxillary anterior region, cortical bone is present not only on the labial/palatal side but also around the incisive canal, and root resorption due to root contact with the bone around the incisive canal has been suggested. ${ }^{5,6}$ However, because root resorption is influenced by various factors such as the magnitude of force ${ }^{7}$ and amount of tooth movement, ${ }^{8}$ it is difficult to rule out other factors and conclude that root contact with the cortical bone around the incisive canal is the cause for resorption. Here we report an orthodontic case involving a 21-year-old female with Angle Class 11, division 1 malocclusion who exhibited significant posttreatment root resorption in the maxillary right central incisor that was caused by contact of the root with the

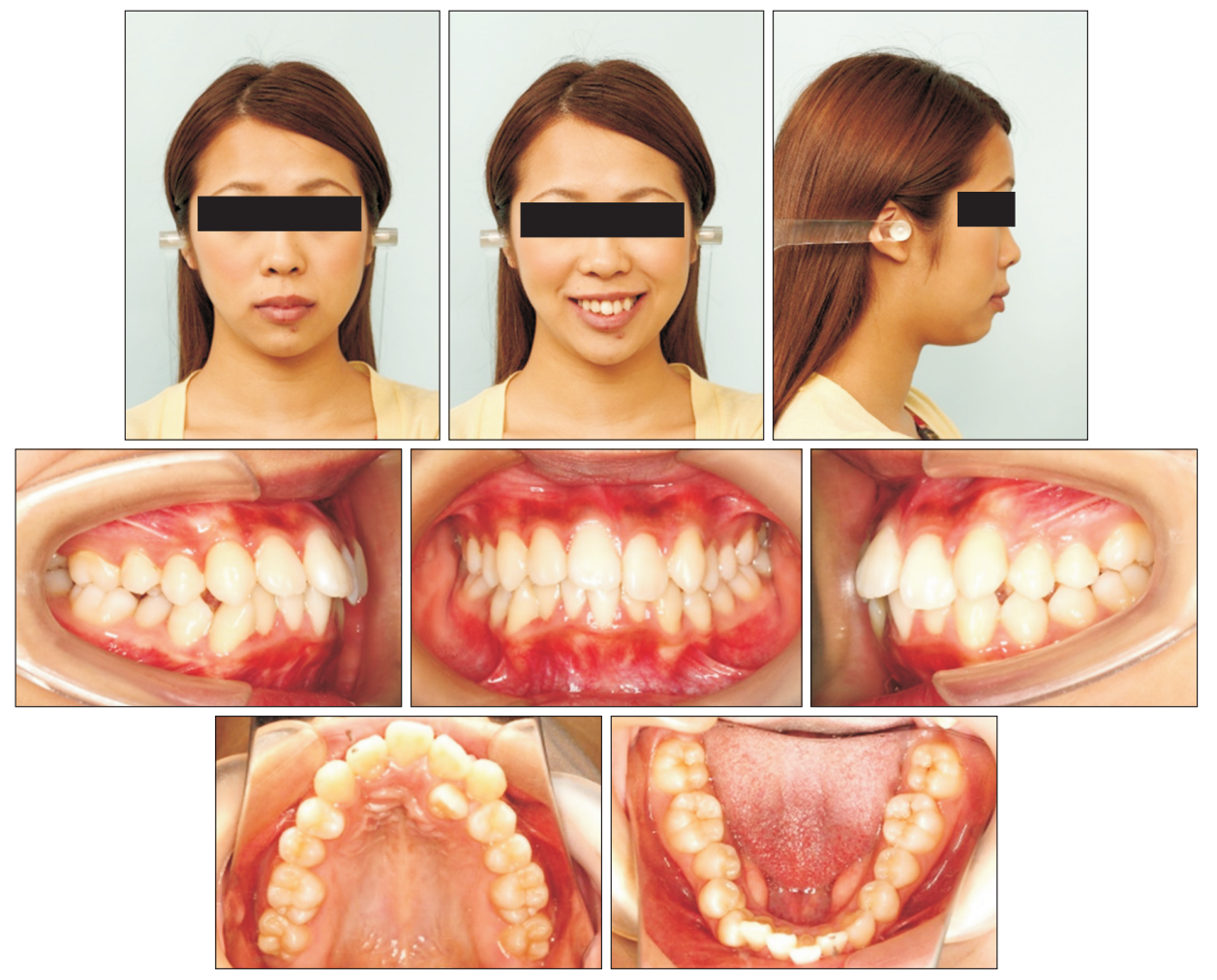

Figure 1. Pretreatment facial and intraoral photographs.
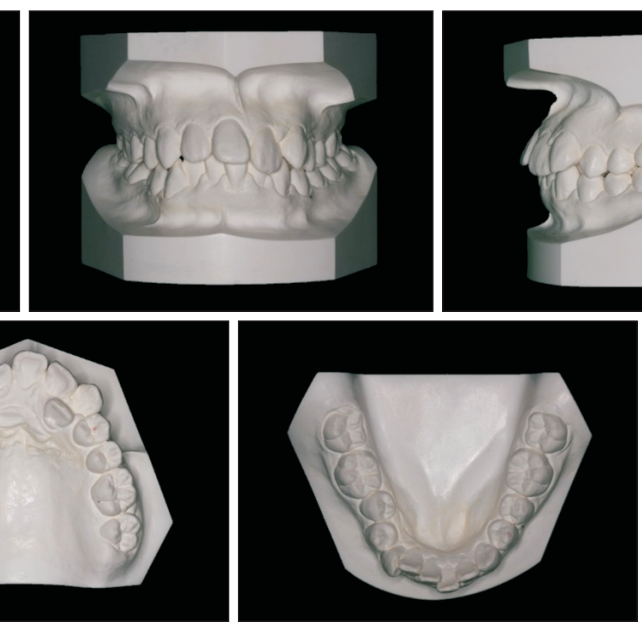
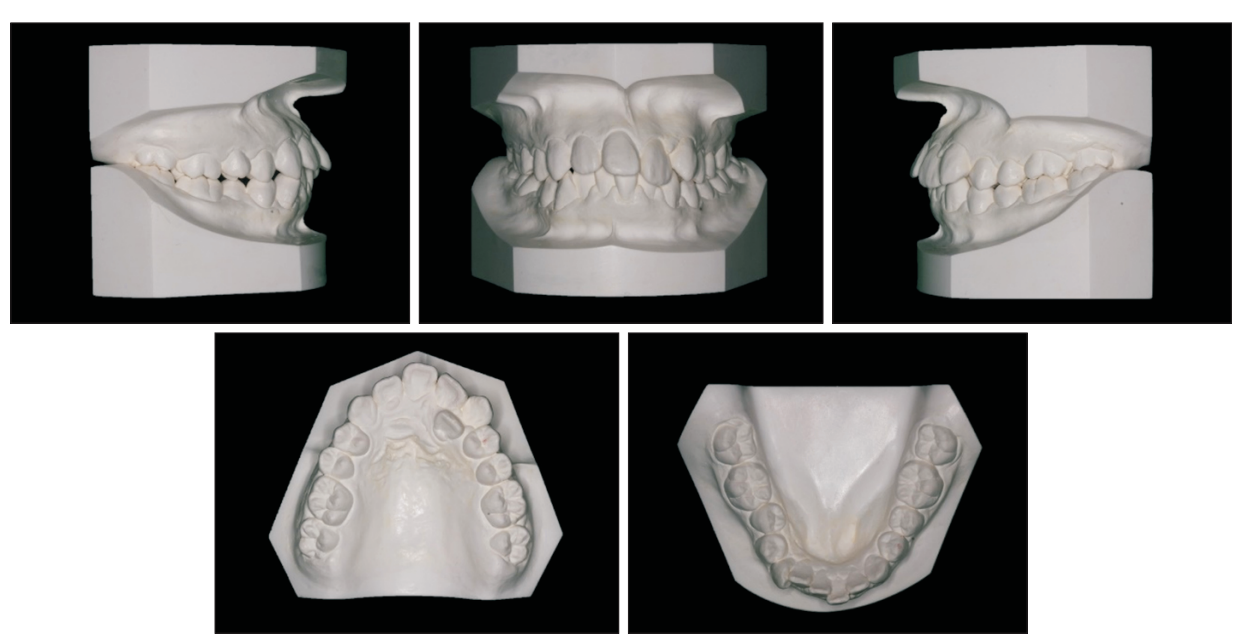
cortical bone around the incisive canal. This report evaluates the relevance of root resorption and root contact with the cortical bone around the incisive canal by performing three-dimensional (3D) cone-beam computed tomography (CBCT) evaluations for the measurement of movement before and after active treatment and preparing 3D models of the maxillary central incisors and the incisive canal with the surrounding bone.

\section{DIAGNOSIS AND ETIOLOGY}

A 21-year-old female presented with a chief complaint of crowding in the maxillary anterior region (Figure 1). She had no history of systemic diseases or allergies. Her dental history was also unremarkable, with no history of trauma to the anterior teeth. Facial photographs showed deviation of the maxillary and mandibular midlines relative to the facial midline; the maxillary midline was deviated to the left by $5.0 \mathrm{~mm}$ and the mandibular midline was deviated to the right by $1.5 \mathrm{~mm}$. The upper and lower lips showed protrusion of 2.4 and $4.0 \mathrm{~mm}$, respectively, from the esthetic line (E-line) (Figure 1).

Model analysis showed Angle Class 1 molar and Class 11 canine relationships with an overjet of $5.3 \mathrm{~mm}$ and overbite of $6.1 \mathrm{~mm}$; lingoversion of the maxillary left lateral incisor, which resulted in crossbite; and arch

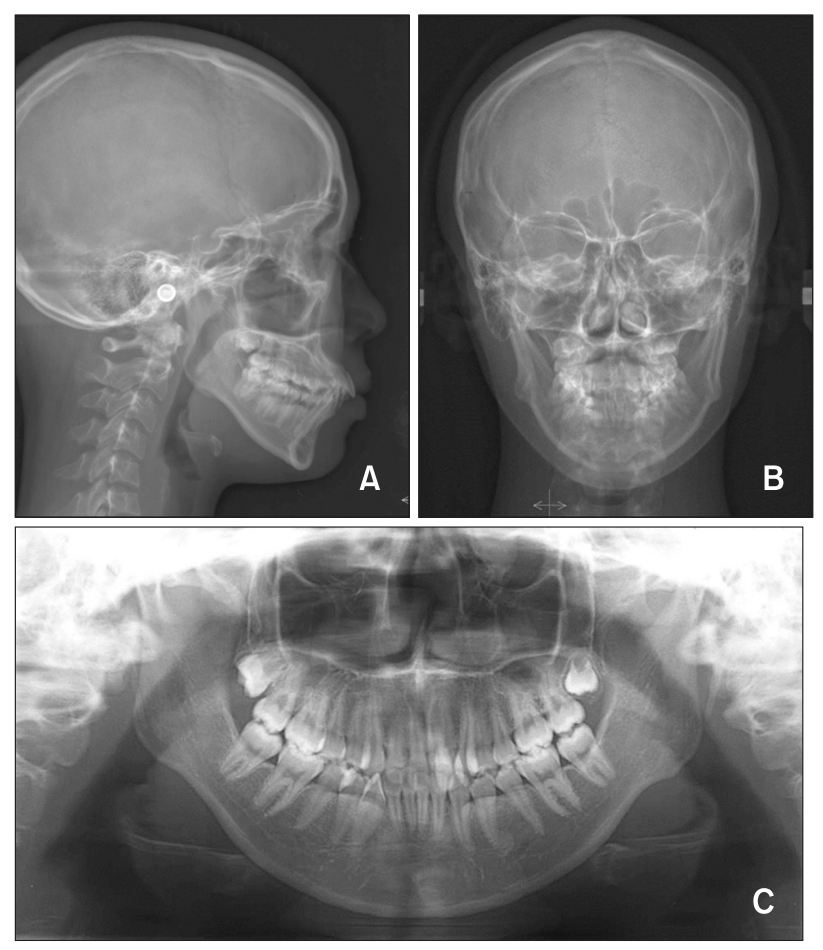

Figure 3. Pretreatment radiographs. A, Lateral cephalogram. B, Posteroanterior cephalogram. C, Panoramic radiograph. length discrepancies of -9.4 and $-5.5 \mathrm{~mm}$ in the maxilla and mandible, respectively (Figures 1 and 2). A panoramic radiograph showed impaction of the bilateral maxillary third molars (Figure 3). Cephalometric analysis demonstrated a Class 1 skeletal relationship (A pointnasion-B point $[\mathrm{ANB}]$ angle, $2.5^{\circ}$ ) and labial inclination of the maxillary incisors (upper incisor to sella-nasion plane angle [U1 to SN], $113.4^{\circ}$; Table 1). The midlines of the maxillary and mandibular incisors observed on a posteroanterior (PA) cephalogram corresponded to those observed during extraoral examination (Figure 3). In addition, CBCT (Finecube; Yoshida, Tokyo, Japan) of the maxillary anterior alveolar region showed a large incisive canal in close proximity to the roots of the maxillary central incisors (Figure 4). We also used the CBCT image to assess the feasibility of using mini-screws and their implantation sites.

\section{TREATMENT OBJECTIVES}

The primary treatment objectives were as follows: to coincide the maxillary and mandibular incisor midlines, eliminate the crossbite by reversing the lingoversion of the maxillary left lateral incisor, normalize the labial inclination of the maxillary anterior teeth, and establish a satisfactory facial profile and occlusion by normalizing the overjet and overbite and achieving Class 1 canine and appropriate molar relationships.

\section{TREATMENT ALTERNATIVES}

We explored three treatment alternatives to meet the treatment objectives.

Table 1. Pre- and post-treatment cephalometric variables with reference values

\begin{tabular}{cccr}
\hline Measurement $\left(^{\mathbf{}}\right.$ ) & Pretreatment & $\begin{array}{c}\text { Post- } \\
\text { treatment }\end{array}$ & Norm $^{\mathbf{9} *}$ \\
\hline SNA & 79.5 & 79.0 & $82.3 \pm 3.5$ \\
SNB & 77.0 & 77.0 & $78.9 \pm 3.5$ \\
ANB & 2.5 & 2.0 & $3.4 \pm 1.8$ \\
FMA & 33.3 & 33.3 & $28.8 \pm 5.2$ \\
U1 to SN & 113.4 & 101.0 & $104.5 \pm 5.6$ \\
IMPA & 85.6 & 83.5 & $96.3 \pm 5.8$ \\
FMIA & 61.1 & 63.2 & $54.6 \pm 6.5$ \\
\hline
\end{tabular}

SNA, Sella-nasion-A point; SNB, sella-nasion-B point; ANB, A point-nasion-B point; FMA, Frankfort mandibular plane angle; U1 to SN, upper incisor to sella-nasion plane angle; IMPA, incisor mandibular plane angle; FMIA, Frankfort mandibular incisor angle.

*Norm indicates normal values \pm standard deviation for a Japanese female. 

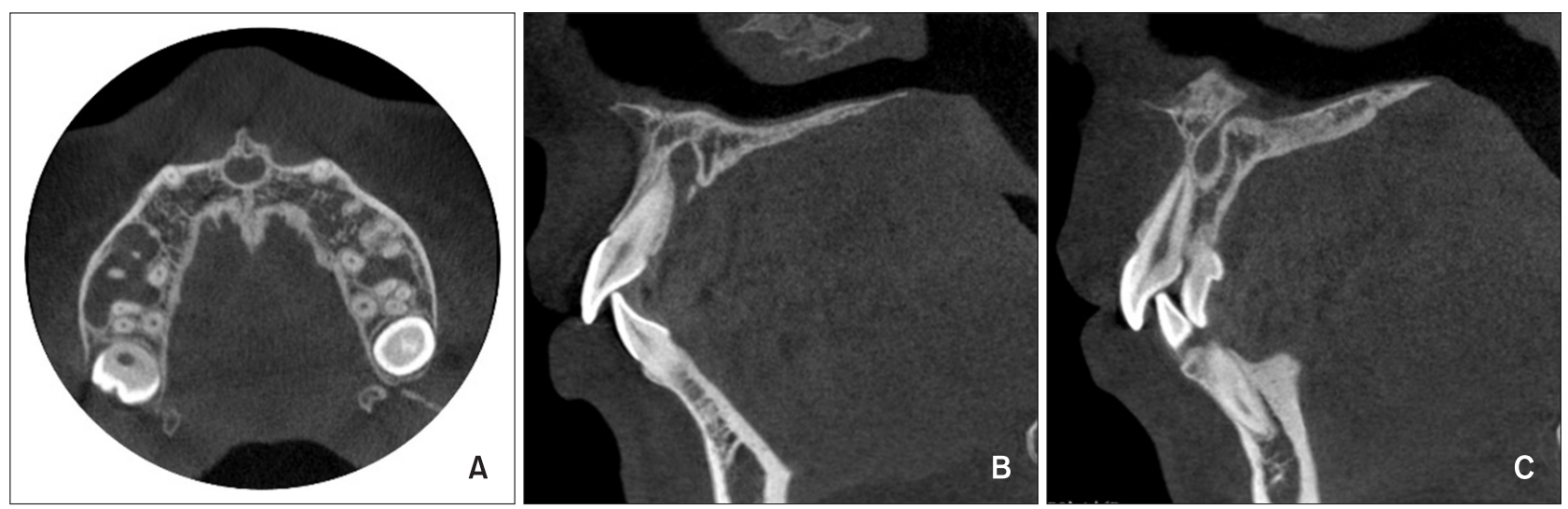

Figure 4. Pretreatment cone-beam computed tomography images. A, Transverse image at the apex level of the maxillary incisor roots. $\mathbf{B}$, Parasagittal image of the maxillary right central incisor. $\mathbf{C}$, Parasagittal image of the maxillary left central incisor.
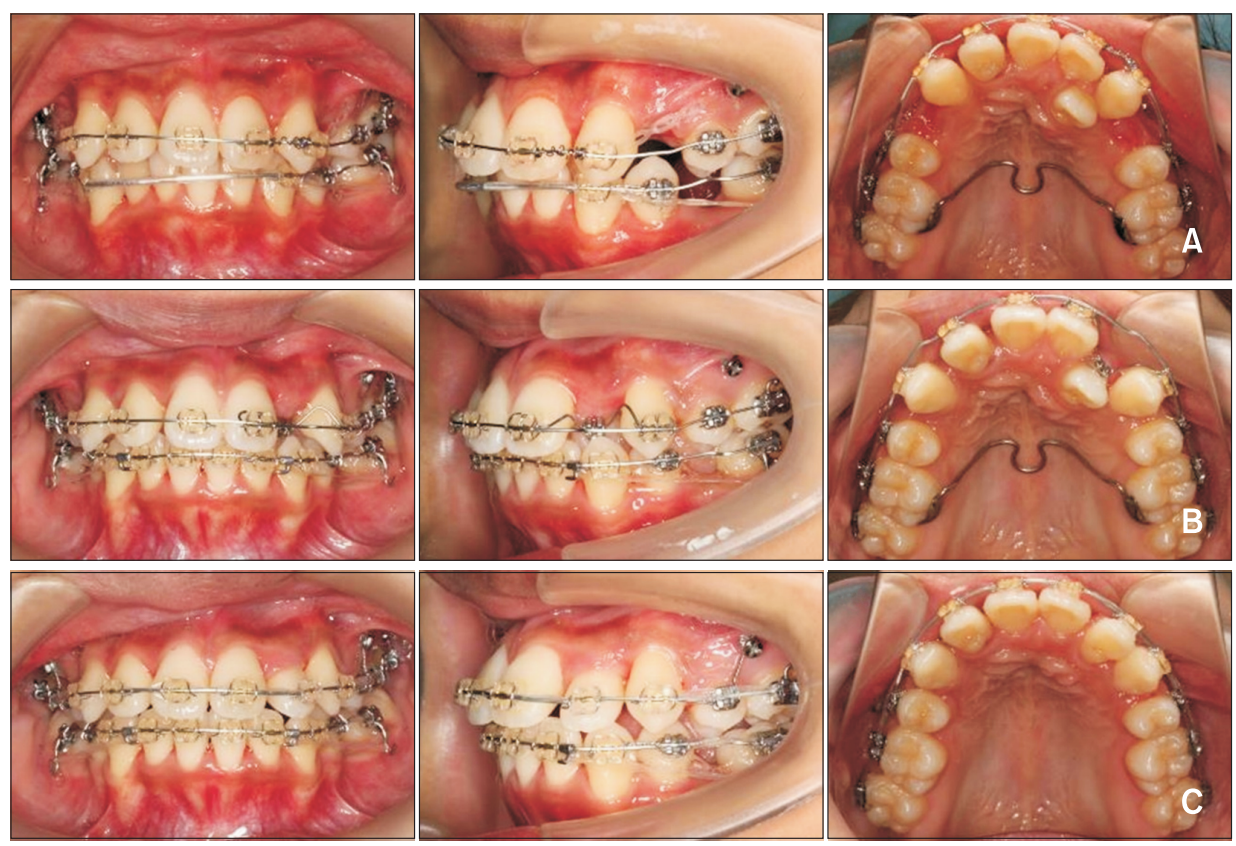

Figure 5. Treatment progress. A, After 1 month of active treatment. B, After 9 months of active treatment. $\mathbf{C}$, After 13 months of active treatment.

The first one involved extraction of the bilateral maxillary first premolars and mandibular second premolars, followed by application of maximum anchorage in the maxilla and moderate anchorage in the mandible for mesialization of the mandibular molars. The second one involved extraction of the bilateral maxillary and mandibular first premolars, followed by application of maximum anchorage in the maxilla. The third one involved extraction of the bilateral maxillary lateral incisors and mandibular first premolars, followed by application of maximum anchorage in the maxilla and moderate anchorage in the mandible for mesialization of the mandibular molars.

All three options involved extraction of the bilateral maxillary third molars, and the first and second options involved the use of mini-screws in the maxilla. Although both options would involve lingual inclination and movement of the maxillary and mandibular incisors, the extent of change would be greater with the second option. The third option had an advantage of a shorter treatment duration achieved by extraction of the maxillary lateral incisor exhibiting lingoversion; however, there were serious concerns regarding the guidance for mandibular lateral movement and esthetics, which could be compromised by differences in the crown morphologies. After extensive discussion with the patient, we selected the first option after considering the arch length discrepancies, incisal axes, and anatomical structure of the 
incisive canal.

\section{TREATMENT PROGRESS}

The bilateral maxillary first premolars and third molars and the bilateral mandibular second premolars were extracted before orthodontic treatment initiation. Preadjusted brackets with 0.018-inch slots (Dentsply Sirona Japan, Tokyo, Japan) were bonded to each tooth, and $0.016 \times 0.022$-inch improved superelastic nickeltitanium (Ni-Ti) archwires improved superelastic nickeltitanium archwires (ISW) (LCtH; Tomy International,
Tokyo, Japan) were used as the working archwires throughout the active treatment period. The long hooks were attached on the brackets of the mandibular first molars to prevent mesial inclination. After 1 month, a mini-screw (Dual Top; Jeil Medical Co., Seoul, Korea) was inserted in the buccal bone between the maxillary second premolars and first molars on both sides, and retraction of the maxillary canines was initiated with ISW incorporating gable bends between the canines and second premolars (Figure 5A). A Ni-Ti open-coil spring (50 g) was simultaneously placed between the maxillary left central incisor and canine for movement of the maxillary

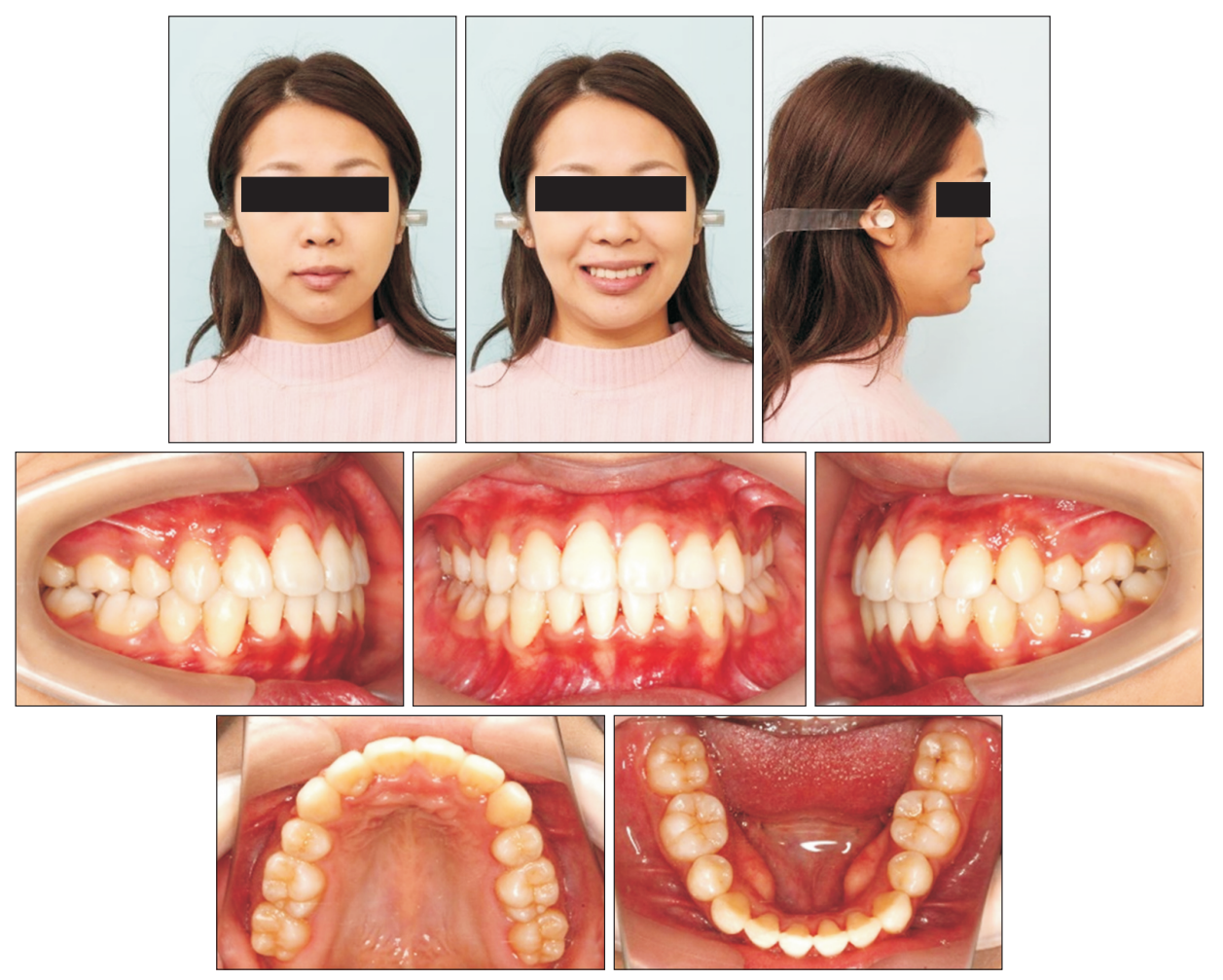

Figure 6. Post-treatment facial and intraoral photographs.
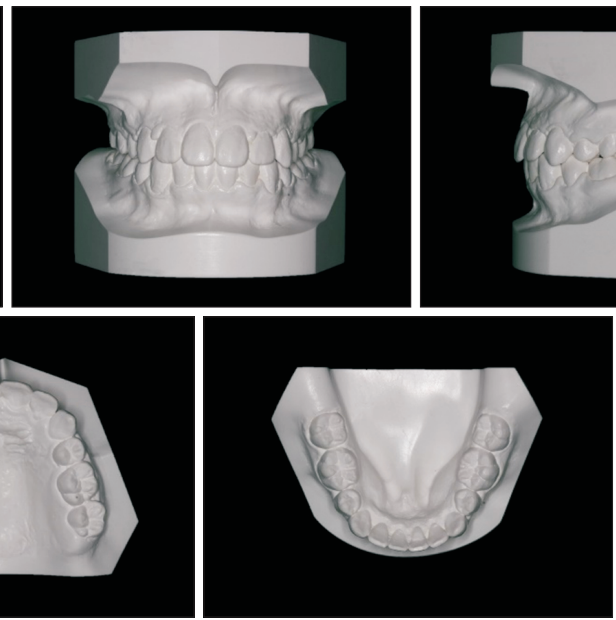
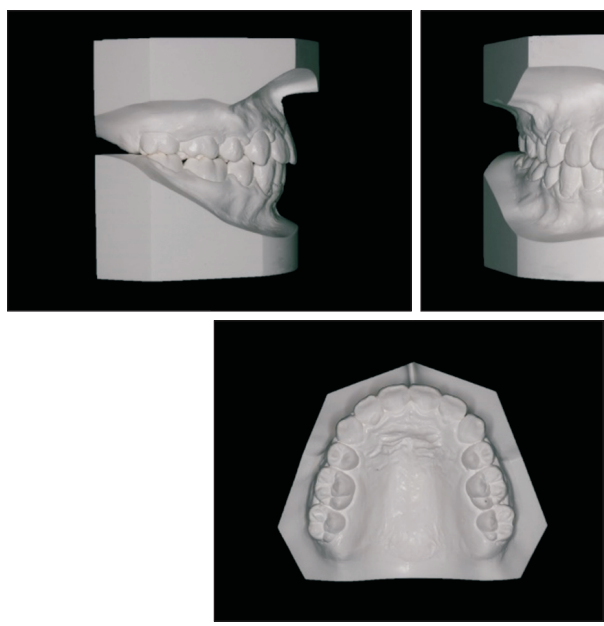
midline to the right. At 3 months, brackets were bonded to the mandibular incisors for leveling with a $0.016 \times$ 0.022-inch ISW. Subsequently, at 9 months, a bracket was bonded to the maxillary left lateral incisor for correction of the lingoversion and crossbite with a $0.016 \times$ 0.022-inch ISW (Figure 5B). At 13 months, maxillary anterior retraction was initiated with a $0.016 \times 0.022$-inch ISW incorporating a compensating curve in the premolar and molar regions (Figure 5C). Subsequently, detailing with Class 11 elastics was initiated at 15 months. After 30 months of active treatment, the maxillary and mandibular incisor midlines coincided and the brackets and mini-screws were removed. Immediately after removal, a circumferential retainer for the maxilla and a Hawley retainer for the mandible were delivered.

\section{RESULTS}

Although the upper lip protruded by $2.4 \mathrm{~mm}$ and the lower lip protruded by $4.0 \mathrm{~mm}$ from the E-line before treatment, the amount of protrusion was decreased to 0.2 and $0.9 \mathrm{~mm}$, respectively. The maxillary and mandibular incisor midlines coincided (Figures 6 and 7). The U1 to $\mathrm{SN}$ angle changed from $113.4^{\circ}$ to $101.0^{\circ}$, the maxillary incisor axis was within the mean normal range, and the Frankfort mandibular incisor angle changed from $61.1^{\circ}$ to $63.2^{\circ}$. Among skeletal parameters, the sella-nasion-A point, sella-nasion-B point, and ANB angles were within the normal ranges, with no clockwise rotation of the mandible ${ }^{9}$ (Figures 8, 9, and Table 1). However, because of space gain for the maxillary left lateral incisor and movement of the maxillary midline to the right, insufficient incisor root parallelism with tilting of the maxillary incisors to the right was observed on a post- treatment panoramic radiograph and PA cephalogram (Figure 10): the angle between the facial and maxillary incisor midline was changed from 12.2 degree to -3.5 degree. Furthermore, the maxillary right central incisor, but not the left one, showed signs of significant root resorption. Therefore, we performed CBCT to confirm the
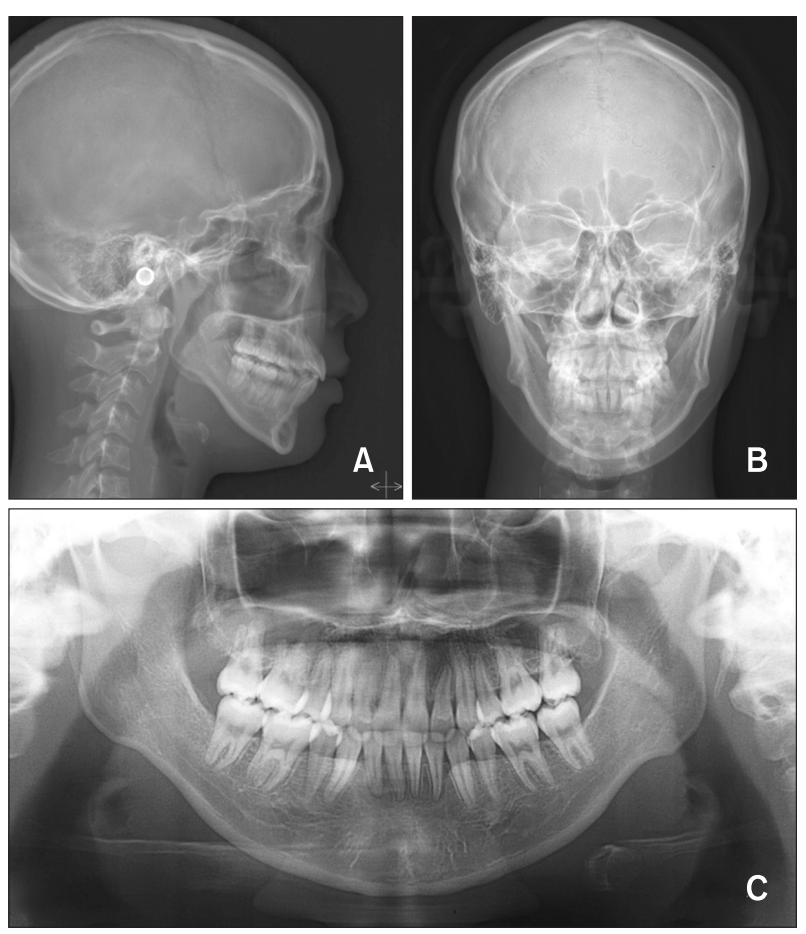

Figure 8. Post-treatment radiographs. A, Lateral cephalogram. B, Posteroanterior cephalogram. C, Panoramic radiograph.

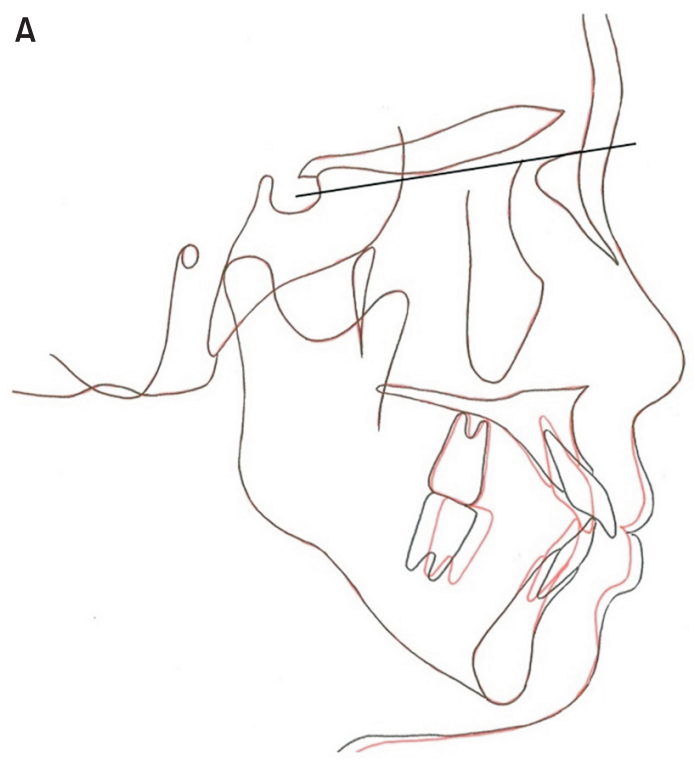

B
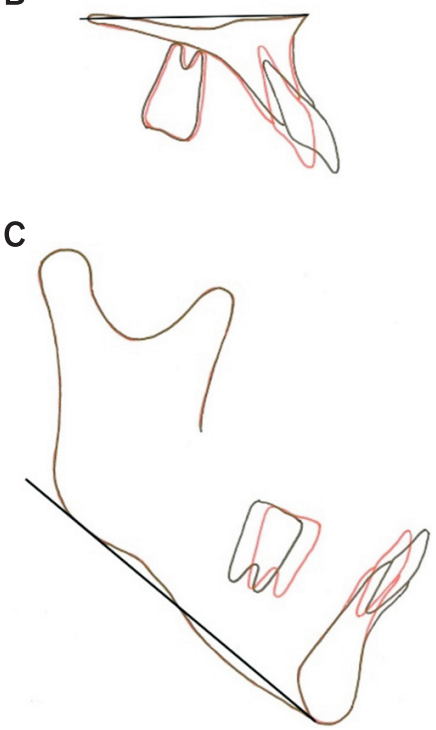

Figure 9. Superimposed tracings of pre- (black line) and post-treatment (red line) lateral cephalograms. A, Superimposition on the sella-nasion plane at the sella. B, Superimposition on the palatal plane at anterior nasal spine. $C_{\text {, }}$ Superimposition on the mandibular plane at the menton. 

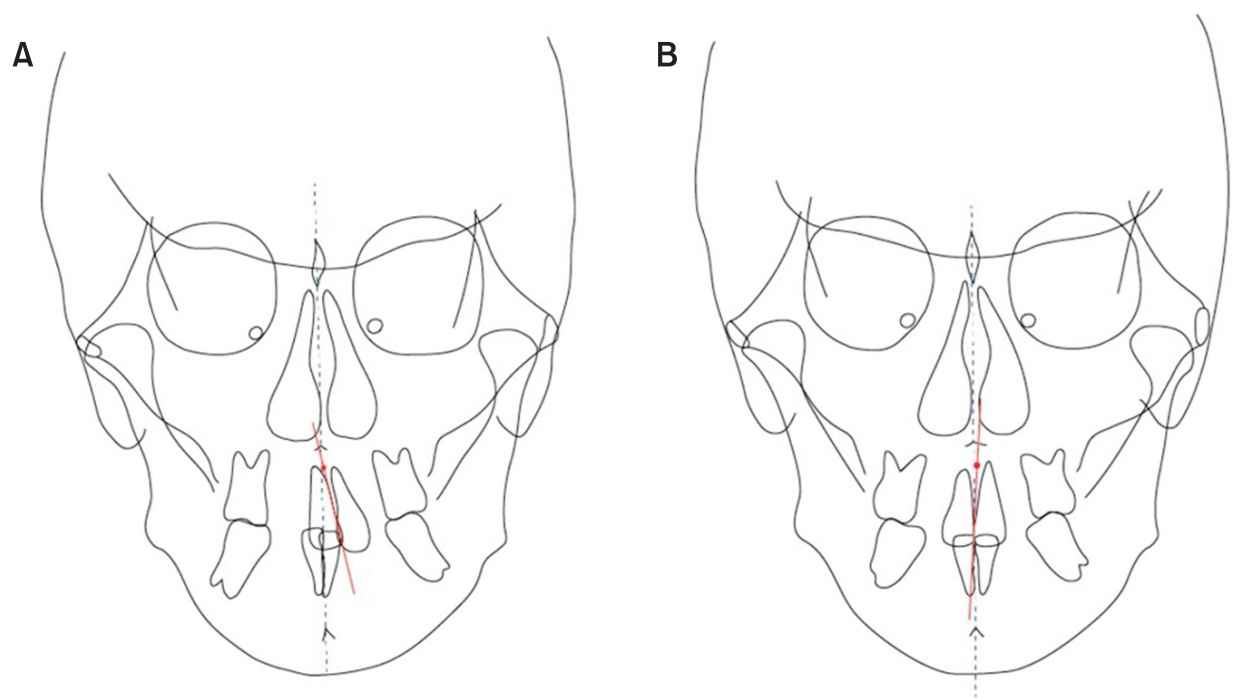

Figure 10. Angle between the maxillary incisor midline (i.e., the line connecting the midpoints of the crowns and root apices of the left and right maxillary central incisors) and the facial midline. A, Before orthodontic treatment. B, After orthodontic treatment.
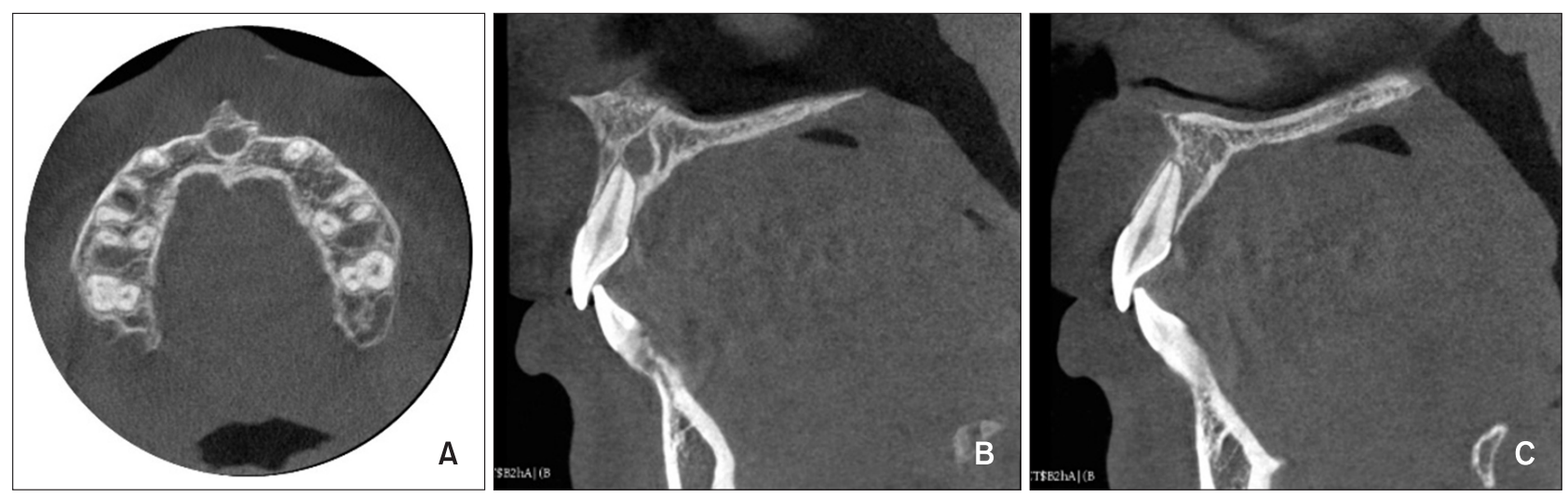

Figure 11. Post-treatment cone-beam computed tomography images. A, Transverse image at the apical level of the maxillary incisor roots. B, Parasagittal image of the maxillary right central incisor. C, Parasagittal image of the maxillary left central incisor.

condition of the incisor roots in three dimensions (Figure 11).

The СВСТ images acquired before and after active treatment were superimposed according to methods reported in a previous study. ${ }^{10}$ In addition, using Mimics software (version 18.0; Materialise, Leuven, Belgium) and 3D slicer (version 4.7.0; www.slicer.org), we confirmed the $3 \mathrm{D}$ configuration, amount of tooth displacement before and after treatment, and amount of root resorption by extracting and creating models of the maxillary central incisors $(1,200-5,000$ Hounsfield unit [HU]), incisive canal ( -800 to $400 \mathrm{HU})$, and cortical bone around the incisive canal (660-2,000 HU; Figures 12 and 13). The periodontal ligament and root apex were relatively clear, and segregation of the root and alveolar bone could be easily performed.

At the time of writing this report, 2 years had passed since the beginning of the retention phase. No remark- able changes were observed in the maxillary anterior region, with maintenance of satisfactory occlusion (Figure 14).

\section{DISCUSSION}

In the present case, a comparison of 3D models created from pre- and post-treatment CBCT images revealed obvious root resorption only in the maxillary right central incisor, with minimal resorption in its left counterpart (Figure 12).

Root resorption can occur in almost all teeth that are orthodontically moved, ${ }^{11}$ although the reason remains unclear. Allergies ${ }^{12}$ and genetic factors ${ }^{13}$ have been considered to be associated with root resorption, in addition to sex, ${ }^{14}$ age, ${ }^{8,15}$ a history of trauma, ${ }^{16}$ the root morphology, ${ }^{8,17,18}$ 3D tooth movement (amount, direction, and even rotation), ${ }^{7,19}$ the magnitude of force, ${ }^{7,8}$ and the 


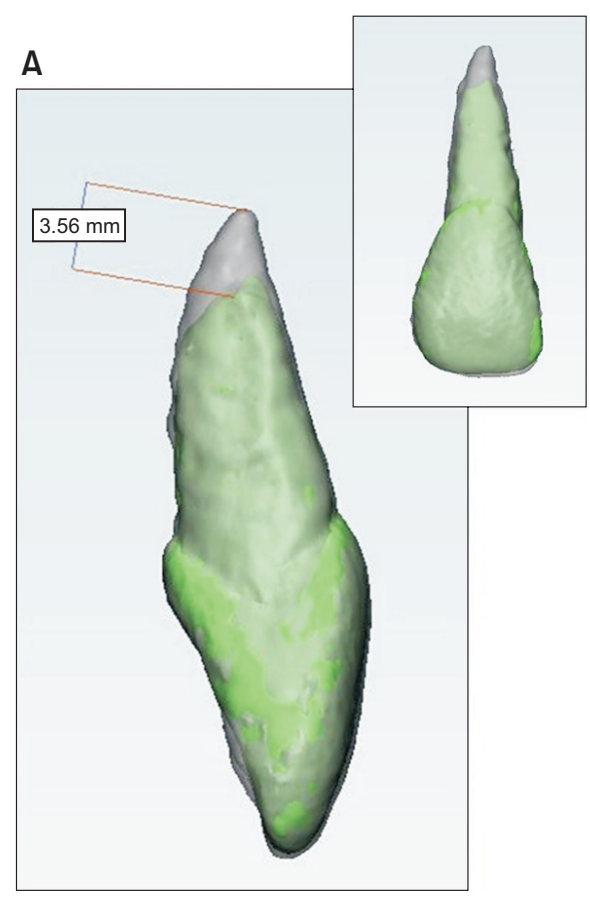

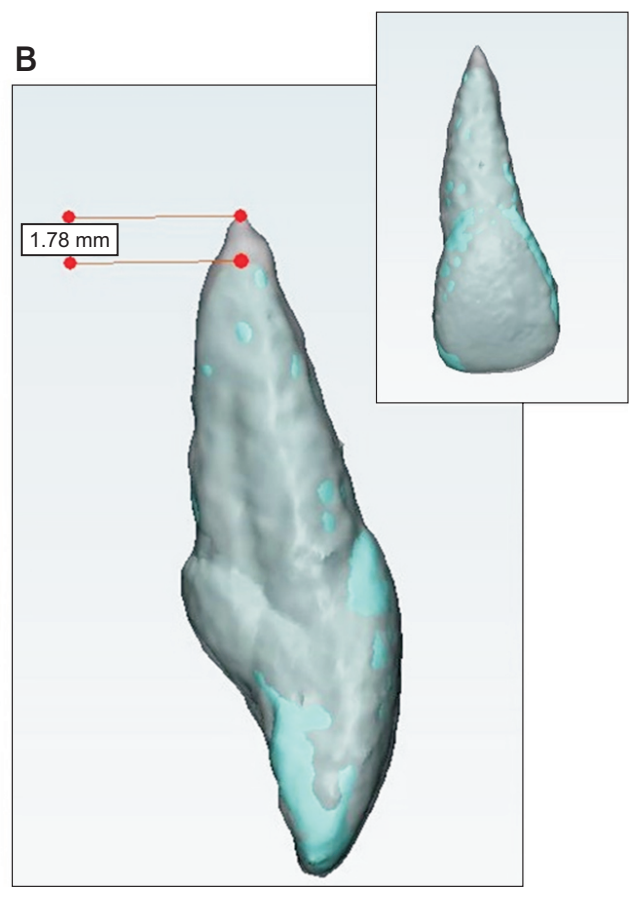

Figure 12. Superimposed three-dimensional models of pre- and post-treatment maxillary central incisors. A, Right. B, Left.

Light blue, Post-treatment maxillary left central incisor; light green, post-treatment maxillary right central incisor; gray, pre-treatment incisors (left and right).

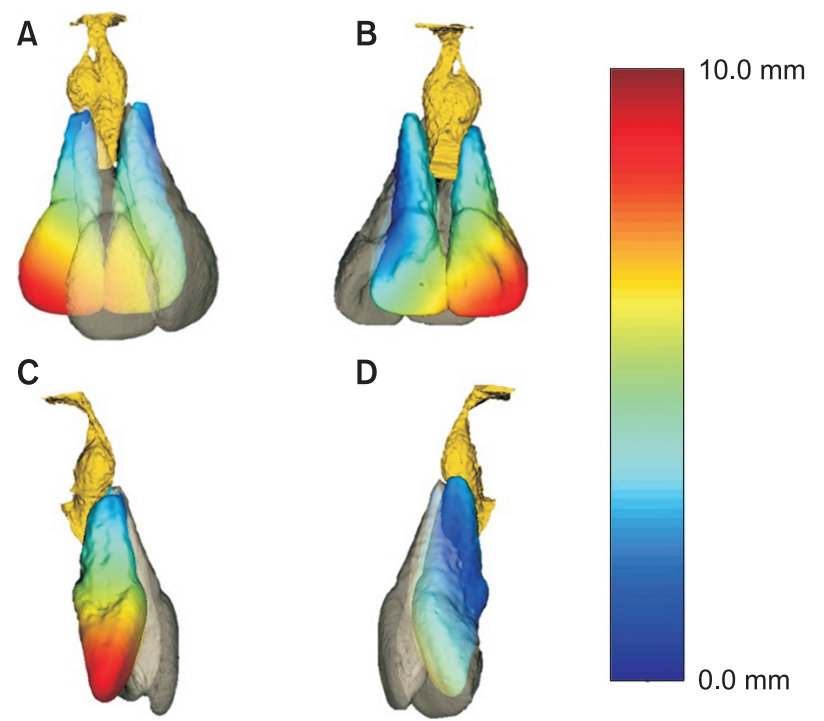

Figure 13. Amount of displacement of the maxillary incisors during active treatment. A color scale is provided to indicate the amount of displacement. A, Frontal view. B, Posterior view. C, Right view. D, Left view.

treatment duration. ${ }^{3,8,10,17}$ In the present case, sagittal displacement of the maxillary anterior teeth was judged to be small on superimposed tracings of pre- and posttreatment lateral cephalograms (Figure 9), whereas the amount of lateral movement observed on PA cephalograms was large (Figure 10). This suggests that accurate two-dimensional (2D) and 3D measurements of movement at various sites of the tooth are important for the evaluation of root resorption. Compared with that in a previous study evaluating 2D images, ${ }^{5,6}$ the relationship between the tooth movement pattern and root resorption in our patient became more evident after 3D reconstruction of the images. We found no significant difference in the amount of apical displacement between the maxillary left and right central incisors (Figure 13 and Table 2). Nonetheless, the amount of root resorption in the maxillary right central incisor was greater than previously reported values (Table 2). ${ }^{11}$ Although the amount of crown movement was greater for the maxillary right central incisor than for the left central incisor, a previous study reported that the amount of lingual inclination of the crown does not significantly influence root resorption. ${ }^{20}$ It is assumed that the amount and direction of root apex movement have a greater influence on root resorption than does the amount of crown movement. Indeed, the findings of several previous studies focusing on root apex movement support this assumption. ${ }^{19}$ Although further studies are necessary, it is unlikely that the root resorption in our patient was primarily caused by the amount and direction of root apex movement.

Another possible cause of root resorption in the present case is the application of intensive force only to the apical segment of the maxillary right central incisor. In this regard, it is desirable to calculate the stress distribution in the root apex. However, we did not choose any force system that applied selective orthodontic forces to the maxillary right central incisor. In fact, right-sided crown movement of the bilateral maxillary incisors during midline correction was accompanied by almost equal 


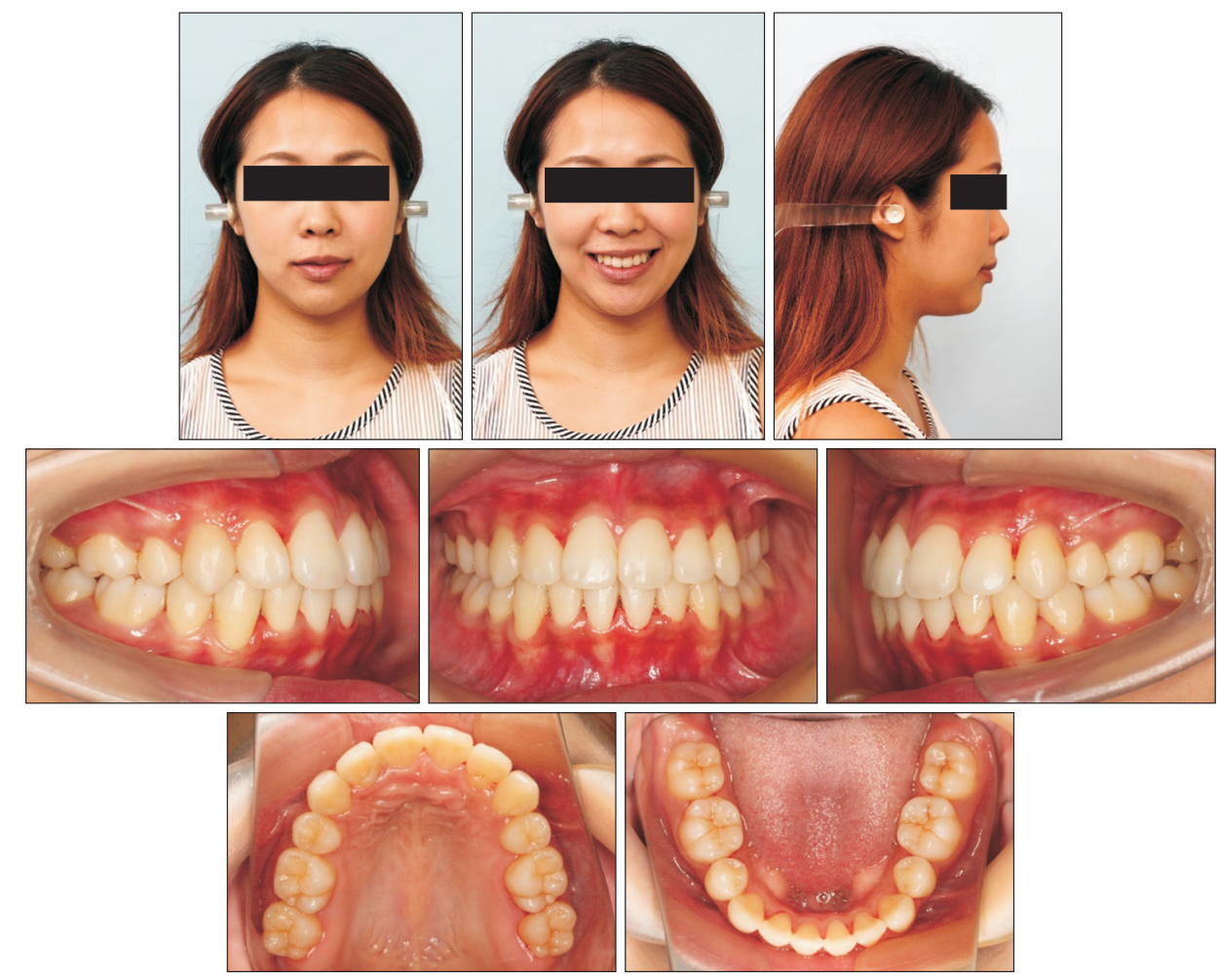

Figure 14. Post-retention facial and intraoral photographs.

Table 2. Apical displacement and root resorption values* for the maxillary central incisors in the present case and previous studies

\begin{tabular}{lccccccc}
\hline \multirow{2}{*}{ Measurement } & \multicolumn{2}{c}{ Present case } & \multirow{2}{*}{ Segal et al. ${ }^{11}$} & & \multicolumn{2}{c}{ Pan and Chen $^{21}$} \\
\cline { 2 - 3 } & Right & Left & & Contact & No contact \\
\hline Displacement (mm) & 2.3 & 3.1 & & $2.4 \pm 0.76$ & & $1.99 \pm 1.74$ & $1.34 \pm 1.79$ \\
Resorption (mm) & 3.6 & 1.8 & & $1.4 \pm 0.45$ & & $2.63 \pm 0.93$ & $1.14 \pm 0.83$ \\
\hline
\end{tabular}

Values are presented as number only or mean \pm standard deviation.

*The values were reported for the maxillary central incisors.

left-sided movement of the root apices (Figure 10). Moreover, when the maxillary incisors were retracted, the root that contacted the cortical bone of the incisive canal showed resorption along the cortical bone (Figures 11-13), with no remodeling observed in the incisive canal (Figure 13). It is assumed that the size and configuration of the incisive canal contributes to root resorption. This notion is further supported by the fact that the difference in the amount of resorption between roots contacting the incisive canal and those without contact in a previous study ${ }^{21}$ was similar to the difference between the left and right central incisors in the present case (Table 2). Previously, Cho et al. ${ }^{6}$ reported that the incisive canal width is larger than the inter-root distance in over $60 \%$ patients. The incisive canal width was significantly larger than the apical inter-root distance in our case. The shape of the incisive canal varies, with cylindrical or conical canals and spindle-shaped canals ${ }^{22}$ accounting for approximately $70 \%$ and $10 \%{ }^{22}$ of all canals, respectively. Our patient exhibited a spindleshaped canal, with the largest diameter exceeding the average apical inter-root distance. Moreover, the distance between the root apex of the maxillary central incisor and the incisive canal was smaller than the average distance reported in other patients (Table 3). ${ }^{6,23,24}$ Taken together, the root apex is more likely to contact the incisive canal if there is no change in the incisive canal itself. Although we set the treatment objectives after confirming the positional relationship between the incisor root and the incisive canal, the improper force system resulted in contact between the two structures during treatment. Collectively, the findings from this case highlight the importance of precise, careful, and thoroughly preplanned control of maxillary incisor movement within 
Table 3. Anatomical relationship between the incisive canal and the root apices

\begin{tabular}{ccc}
\hline Measurement & Present case & Norm \\
\hline Diameter (mm) & 7.0 & $3.5 \pm 1.1^{23}$ \\
& & $3.5 \pm 0.9^{6}$ \\
$\begin{array}{c}\text { Distance between the } \\
\text { root apices (mm) }\end{array}$ & 5.2 & $7.1 \pm 0.84^{6}$ \\
$\begin{array}{c}\text { Distance from the } \\
\text { root apex (mm) }\end{array}$ & 0.6 (right) & $4.0 \pm 1.5^{24}$ \\
\hline
\end{tabular}

Values are presented as number only or mean \pm standard deviation. The superscripts in the Norm column indicate the corresponding reference number.

an anatomically acceptable range.

\section{CONCLUSION}

In conclusion, we described a case involving different degrees of root resorption in the bilateral maxillary central incisors despite comparable degrees of orthodontic movement on both sides. This phenomenon occurred even after treatment was performed in accordance with a treatment plan based on comprehensive analyses, including CBCT studies. The different positional relationship (including contact) between the incisor root and the incisive canal may have played a role in the different root resorption patterns; however, further investigation is necessary to confirm this assumption.

\section{CONFLICTS OF INTEREST}

No potential conflict of interest relevant to this article was reported.

\section{REFERENCES}

1. Weltman B, Vig KW, Fields HW, Shanker S, Kaizar EE. Root resorption associated with orthodontic tooth movement: a systematic review. Am J Orthod Dentofacial Orthop 2010;137:462-76; discussion 12A.

2. Li F, Hu HK, Chen JW, Liu ZP, Li GF, He SS, et al. Comparison of anchorage capacity between implant and headgear during anterior segment retraction: a systematic review. Angle Orthod 2011;81:915-22.

3. Otis LL, Hong JS, Tuncay OC. Bone structure effect on root resorption. Orthod Craniofac Res 2004; 7:165-77.

4. Horiuchi A, Hotokezaka H, Kobayashi K. Correlation between cortical plate proximity and apical root resorption. Am J Orthod Dentofacial Orthop 1998;114:311-8.
5. Chung CJ, Choi YJ, Kim KH. Approximation and contact of the maxillary central incisor roots with the incisive canal after maximum retraction with temporary anchorage devices: report of 2 patients. Am J Orthod Dentofacial Orthop 2015;148:493502.

6. Cho EA, Kim SJ, Choi YJ, Kim KH, Chung CJ. Morphologic evaluation of the incisive canal and its proximity to the maxillary central incisors using computed tomography images. Angle Orthod 2016;86:571-6.

7. Roscoe MG, Meira JB, Cattaneo PM. Association of orthodontic force system and root resorption: a systematic review. Am J Orthod Dentofacial Orthop 2015;147:610-26.

8. Fox N. Longer orthodontic treatment may result in greater external apical root resorption. Evid Based Dent 2005;6:21.

9. lizuka T, lshikawa F. Normal standards for various cephalometric analysis in Japanese adult. J Stomatol Soc 1958;25:260-72.

10. Park JH, Tai K, Owtad P. 3-Dimensional cone-beam computed tomography superimposition: a review. Semin Orthod 2015;21:263-73.

11. Segal GR, Schiffman PH, Tuncay OC. Meta analysis of the treatment-related factors of external apical root resorption. Orthod Craniofac Res 2004;7:71-8.

12. Owman-Moll P, Kurol J. Root resorption after orthodontic treatment in high- and low-risk patients: analysis of allergy as a possible predisposing factor. Eur J Orthod 2000;22:657-63.

13. Nieto-Nieto N, Solano JE, Yañez-Vico R. External apical root resorption concurrent with orthodontic forces: the genetic influence. Acta Odontol Scand 2017;75:280-7.

14. Baumrind S, Korn EL, Boyd RL. Apical root resorption in orthodontically treated adults. Am J Orthod Dentofacial Orthop 1996;110:311-20.

15. Bishara SE, Vonwald L, Jakobsen JR. Changes in root length from early to mid-adulthood: resorption or apposition? Am J Orthod Dentofacial Orthop 1999;115:563-8.

16. Malmgren 0, Goldson L, Hill C, Orwin A, Petrini $\mathrm{L}$, Lundberg M. Root resorption after orthodontic treatment of traumatized teeth. Am J Orthod 1982;82:487-91.

17. Sameshima GT, Sinclair PM. Characteristics of patients with severe root resorption. Orthod Craniofac Res 2004;7:108-14.

18. Lee RY, Årtun J, Alonzo TA. Are dental anomalies risk factors for apical root resorption in orthodontic patients? Am J Orthod Dentofacial Orthop 1999;116:187-95.

19. Han G, Huang S, Von den Hoff JW, Zeng X, Kui- 
jpers-Jagtman AM. Root resorption after orthodontic intrusion and extrusion: an intraindividual study. Angle Orthod 2005;75:912-8.

20. Parker RJ, Harris EF. Directions of orthodontic tooth movements associated with external apical root resorption of the maxillary central incisor. Am J Orthod Dentofacial Orthop 1998;114:677-83.

21. Pan Y, Chen S. Contact of the incisive canal and upper central incisors causing root resorption after retraction with orthodontic mini-implants: A CBCT study. Angle Orthod 2019;89:200-5.

22. Thakur AR, Burde K, Guttal K, Naikmasur VG. Anatomy and morphology of the nasopalatine canal us- ing cone-beam computed tomography. Imaging Sci Dent 2013;43:273-81.

23. Al-Amery SM, Nambiar P, Jamaludin M, John J, Ngeow WC. Cone beam computed tomography assessment of the maxillary incisive canal and foramen: considerations of anatomical variations when placing immediate implants. PLoS One 2015;10: e0117251.

24. Matsumura T, Ishida Y, Kawabe A, Ono T. Quantitative analysis of the relationship between maxillary incisors and the incisive canal by cone-beam computed tomography in an adult Japanese population. Prog Orthod 2017;18:24. 